\title{
Evaluation de Technologies de Substrats Céramiques sous des Cyclages en Température de Forte Amplitude
}

\author{
Laurent Dupont ${ }^{(1,2)}$, Zoubir Khatir ${ }^{(1)}$, Stéphane Lefebvre ${ }^{(2)}$, Serge Bontemps ${ }^{(3)}$, Régis Meuret ${ }^{(4)}$ \\ (1) INRETS LTN, 2 av. du Général Malleret-Joinville, F94114 Arcueil \\ (2) SATIE, UMR CNRS 8029, ENS de Cachan 61 Av. du président Wilson F94235 Cachan \\ (3) MICROSEMI-APT Europe, Chemin de Magret, 33700 Merignac \\ (4) HISPANO-SUIZA, Rond Point René Ravaud- Réau, F77550 Moissy-Cramayel
}

\begin{abstract}
RÉSUMÉ. Cette étude cherche à évaluer le comportement de substrats céramiques, utilisés pour la réalisation de modules de puissance, pour des applications aéronautiques, à haute température, et sous des cycles de température de forte amplitude. L'article présente les résultats expérimentaux obtenus sur des substrats céramiques de différentes technologies. Plusieurs véhicules de test ont été réalisés par la société MICROSEMI-APT Europe. Ils sont composés de différents substrats céramiques, assemblés par brasage sur des semelles $\mathrm{AlSiC}$, dont des $\mathrm{DCB}$ AlN et $\mathrm{Al}_{2} \mathrm{O}_{3}$, ainsi que des solutions de technologie $\mathrm{AMB}$ comme le $D A B$ (aluminium sur céramique AlN) ou des substrats $\mathrm{Si}_{3} \mathrm{~N}_{4}$. Cet article montre les modes de défaillances, et compare les différentes technologies dans le but d'identifier les principaux facteurs permettant d'améliorer la durée de vie des assemblages de puissance dans des environnements thermiques sévères.
\end{abstract}

MOTS-CLÉs : Haute température, Modèle thermomécanique, Substrat céramique, Modes de défaillance, Cyclage thermique, Durée de vie.

ABSTRACT. Objective of the study is the evaluation of ceramic substrates used in power modules for aeronautic applications in high temperature with thermal cycles of great amplitude. This article presents experimental results carried out under such severe conditions for different technological ceramic substrates. Several test vehicles have been realised by MICROSEMI-APT Europe Company. They include various ceramic materials soldered on AlSiC base plate, of which DCB with AlN and Al2O3 ceramic substrates with and without dimples and different copper thickness, and AMB substrates with Si3N4 and $D A B$ (aluminium on ceramic). This article shows the failure modes and compare several technologies in order to identify the main factors that could increase the lifetime expectancy of power modules in such harsh environments.

KEYWORDS: High temperature, Thermo-mechanical model, Ceramic substrate, Failure modes, Thermal cycling, Lifetime duration.

Revue. Volume $\mathrm{X}-\mathrm{n}^{\circ} \mathrm{x}$ /année, pages 1 à $\mathrm{X}$ 


\section{Introduction}

Dans différents domaines d'applications (aéronautique, forage, automobile...), nous pouvons observer une augmentation de la demande pour des dispositifs d'électronique de puissance adaptés à un fonctionnement à haute température. Dans ces domaines d'applications, les composants et l'assemblage peuvent subir des contraintes environnementales sévères. Notamment pour des applications avionique, le convertisseur peut être placé sur le réacteur conduisant, dans le pire des cas, à des cycles thermiques entre $-55^{\circ} \mathrm{C}$ et $+200^{\circ} \mathrm{C}$ avec des variations de température de l'ordre de $\pm 10^{\circ} \mathrm{C} / \mathrm{min}$. Afin d'évaluer le possible fonctionnement des assemblages de puissance dans un environnement à haute température, et sa fiabilité lors de cyclage thermique de grande amplitude, des essais de cyclage thermique ont été effectués sur différents véhicules de test. Ces véhicules de test, réalisés par la société MICROSEMI-APT Europe, se composent de différents substrats céramiques brasés sur des semelles AlSiC. Les résultats expérimentaux doivent permettre d'identifier les modes de défaillances, et nous aider à proposer des solutions afin d'améliorer la durée de vie des modules de puissance, en accord avec le profil de mission imposée. De plus, nous avons également pour objectif d'identifier les principaux facteurs susceptibles d'accroître la durée de vie des modules de puissance dans un environnement aussi sévère que celui présenté ci-dessus. Cet article se focalise sur les modes de défaillances observés lors de cycles thermiques de grande amplitude à haute température et plus précisément, sur la fissure conchoïdale de la céramique sous les métallisations en cuivre des substrats DCB, ainsi que la délaminage de la brasure entre substrat céramique et semelle AlSiC.

\section{Présentation des véhicules de test}

Afin de minimiser les différences entre les coefficients de dilatation thermiques (CDT), entre les substrats céramiques et la semelle, les véhicules de test sont tous assemblés sur une semelle AlSiC. Les substrats étudiés sont brasés sur la semelle avec le même alliage haute température $\left(\mathrm{T}_{\text {liquidus }}>290^{\circ} \mathrm{C}\right)$. Onze véhicules de test, avec des substrats de technologies différentes, ont été réalisés par MICROSEMI-APT Europe puis intégrés dans le plan d'expérimentation :

- DCB (Direct Copper Bonding) sur céramique $\mathrm{Al}_{2} \mathrm{O}_{3}: 5$ véhicules de test différents dont les céramiques et les métallisations, avec ou sans dimples, sont de différentes épaisseurs.

- DCB sur céramique AlN : 4 véhicules de test dont les métallisations en cuivre, avec et sans dimples, sont de différentes épaisseurs.

- AMB (Active Metal Brazing) sur céramique $\mathrm{Si}_{3} \mathrm{~N}_{4}$ : un véhicule de test avec une épaisseur de cuivre de $400 \mu \mathrm{m}$.

- DAB (Direct Aluminium Bonding) sur céramique AlN : un véhicule de test avec une métallisation en aluminium.

Le tableau 1 présente les principales caractéristiques des différents véhicules de test (VT). Le tableau 2 fait la synthèse des propriétés mécaniques et thermiques des matériaux constituant les différents échantillons. La figure 1 présente trois des différents véhicules de test. 


\section{Protocoles de test}

Afin d'évaluer l'aptitude d'un assemblage de puissance à fonctionner dans un environnement à haute température d'une part, et d'autre part, sa durée de vie lorsqu'il est soumis à des cycles thermiques importants, des cyclages thermiques ont été appliqués en adéquation avec le profil de mission de l'application. Le profil thermique imposé aux véhicules de test est présenté ci-après, avec une durée par cycle d'environ 2 heures, et des taux de variations de la température de l'ordre de $12^{\circ} \mathrm{C} / \mathrm{min}$. La température évolue entre $-30^{\circ} \mathrm{C}$ et $+180^{\circ} \mathrm{C}$ avec une durée de palier de 30min afin de prendre en compte le phénomène de fluage de la brasure à température constante. Les tests ont été réalisés dans une enceinte climatique dans les locaux du LTN à l'INRETS. La figure 2 montre l'implantation des véhicules de test dans l'enceinte climatique, ainsi que l'évaluation de l'homogénéité de la température, qui est relevée grâce à six thermocouples implantés dans les semelles de quelques un des véhicules de test.

Les cycles thermiques, présentés dans la figure 3 (variation de la température entre $-30^{\circ} \mathrm{C}$ et $+180^{\circ} \mathrm{C}$ à $\pm 12^{\circ} \mathrm{C} / \mathrm{min}$ avec des paliers de $30 \mathrm{~min}$ ), sont appliqués simultanément à l'ensemble des véhicules de test. Le cyclage thermique est périodiquement interrompu afin de mesurer les indicateurs de vieillissement permettant de détecter l'apparition des modes de défaillance. Ainsi, l'évaluation du vieillissement des brasures est réalisée grâce à l'analyse par microscopie acoustique, avant d'entreprendre le cyclage, puis régulièrement au cours du test. L'intégrité des substrats céramiques a été évaluée par une mesure de la capacité des substrats céramiques (Figure 4) afin de détecter le décollement de la métallisation, tel que décrit dans [3, 5]. L’indicateur de défaillance est arbitrairement fixé pour une variation de $2 \%$ de la capacité initiale, mesurée avant d'entreprendre le cyclage.

Tableau 1. Description des véhicules de test.

\begin{tabular}{|c|l|c|c|c|c|}
\hline Echantillon & \multicolumn{1}{|c|}{ Epaisseur des métallisations } & $\begin{array}{c}\text { Nombre de } \\
\text { substrats }\end{array}$ & $\begin{array}{c}\text { Nature } \\
\text { céramique }\end{array}$ & \multicolumn{2}{c|}{$\begin{array}{c}\text { Géométrie de la céramique } \\
\text { Epaisseur }(\mu \mathrm{m})\end{array}$} \\
\hline VT 1 & $\mathrm{Cu}: 300 \mu \mathrm{m}$ & 4 & $\mathrm{Al}_{2} \mathrm{O}_{3}$ & 380 & $2,54 \times 3,70$ \\
\hline VT 2 & $\mathrm{Cu}: 300 \mu \mathrm{m}$ barrière Ni Dimples & 4 & $\mathrm{Al}_{2} \mathrm{O}_{3}$ & 380 & $2,54 \times 2,70$ \\
\hline VT 3 & $\mathrm{Cu}: 300 \mu \mathrm{m}$ & 4 & $\mathrm{AlN}$ & 635 & $2,54 \times 3,70$ \\
\hline VT 4 & $\mathrm{Cu}: 300 \mu \mathrm{m}$ & 6 & $\mathrm{AlN}$ & 635 & $3,00 \times 1,15$ \\
\hline VT 5 & $\mathrm{Cu}: 400 \mu \mathrm{m}$ barrière Ni & 1 & $\mathrm{Si}_{3} \mathbf{N}_{4}$ & 320 & $3,80 \times 5,10$ \\
\hline VT 6 & $\mathrm{Al}: 400 \mu \mathrm{m}$ barrière Ni & 2 & $\mathrm{AlN}$ & 635 & $3,80 \times 5,80$ \\
\hline VT 7 & $\mathrm{Cu}: 127 \mu \mathrm{m}$ & 8 & $\mathrm{AlN}$ & 635 & $2,54 \times 2,54$ \\
\hline VT 8 & $\mathrm{Cu}: 200 \mu \mathrm{m}$ & 8 & $\mathrm{AlN}$ & 635 & $2,54 \times 2,54$ \\
\hline VT 9 & $\mathrm{Cu}: 127 \mu \mathrm{m}$ & 8 & $\mathrm{Al}_{2} \mathrm{O}_{3}$ & 380 & $2,54 \times 2,54$ \\
\hline VT 10 & $\mathrm{Cu}: 200 \mu \mathrm{m}$ & 8 & $\mathrm{Al}_{2} \mathrm{O}_{3}$ & 380 & $2,54 \times 2,54$ \\
\hline VT 11 & $\mathrm{Cu}: 127 \mu \mathrm{m}$ & $\mathrm{Al}_{2} \mathrm{O}_{3}$ & 500 & $2,54 \times 2,54$ \\
\hline
\end{tabular}


Tableau 2. Principales propriétés physiques des matériaux constituants les véhicules de test.

\begin{tabular}{|l|c|c|c|c|c|c|c|}
\cline { 2 - 7 } \multicolumn{1}{c|}{} & $\boldsymbol{A l N}$ & $\mathrm{Al}_{2} \mathrm{O}_{3}$ & Si3N4 & $\mathbf{C u}$ & $\boldsymbol{A l}$ & AlSiC & $\begin{array}{c}\text { Brasure haute } \\
\text { température }\end{array}$ \\
\hline $\begin{array}{l}\text { Conductivité thermique } \\
(\mathrm{W} / \mathrm{m} . \mathrm{K})\end{array}$ & $170-260$ & $20-30$ & 60 & 390 & 240 & $170-200$ & 23 \\
\hline $\begin{array}{l}\text { CDT } \\
\left(10^{-6} /{ }^{\circ} \mathrm{C}\right)\end{array}$ & $4,2-4,5$ & $7,5-8,1$ & $2-3,4$ & 16 & 23 & $6,5-8$ & 29 \\
\hline $\begin{array}{l}\text { Module de Young } \\
(\mathrm{GPa})\end{array}$ & $300-310$ & $300-380$ & 300 & 130 & 70 & $200-250$ & $24.1-28 \times 10^{-3} \mathrm{~T}$ \\
\hline $\begin{array}{l}\text { Limite mécanique } \\
\text { (MPa) }\end{array}$ & $300-500$ & $250-300$ & 700 & & & & \\
\hline $\begin{array}{l}\text { Seuil de plasticité } \\
\text { (MPa) }\end{array}$ & & & & 70 & 20 & & \\
\hline
\end{tabular}

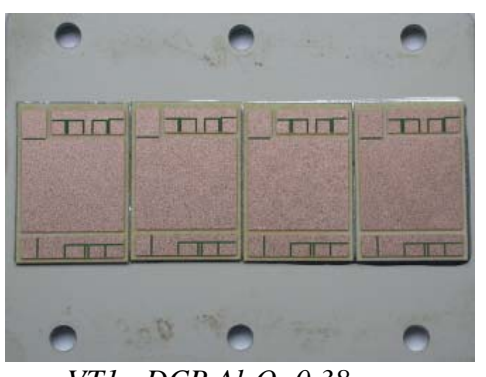

VT1 : $\mathrm{DCB} \mathrm{Al}_{2} \mathrm{O}_{3} 0,38 \mathrm{~mm}$; $\mathrm{Cu} 300 \mu \mathrm{m}$ sans dimples.

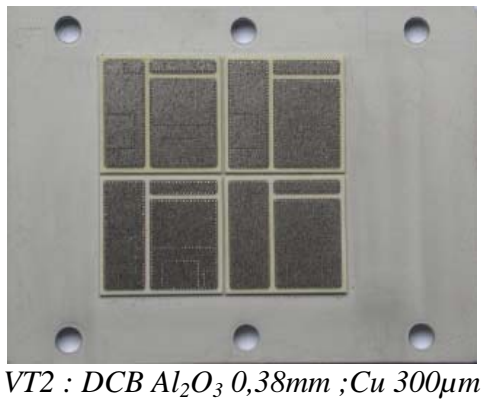
avec dimples.

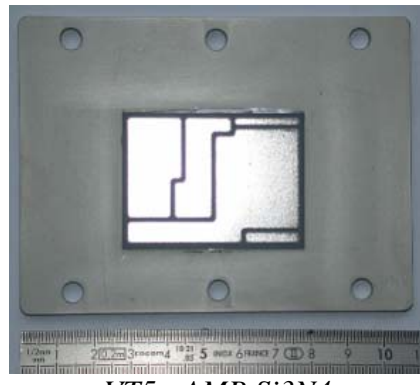

VT5 : AMB Si3N4

Figure 1. Photographie de quelques véhicules de test.

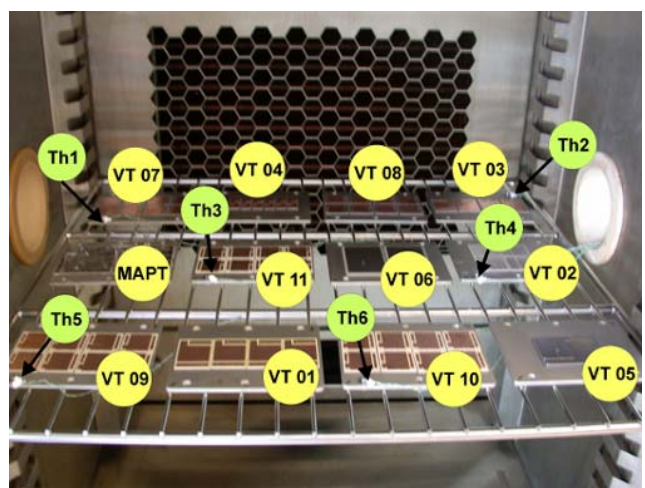

Figure 2. Implantation des véhicules de test dans l'enceinte 


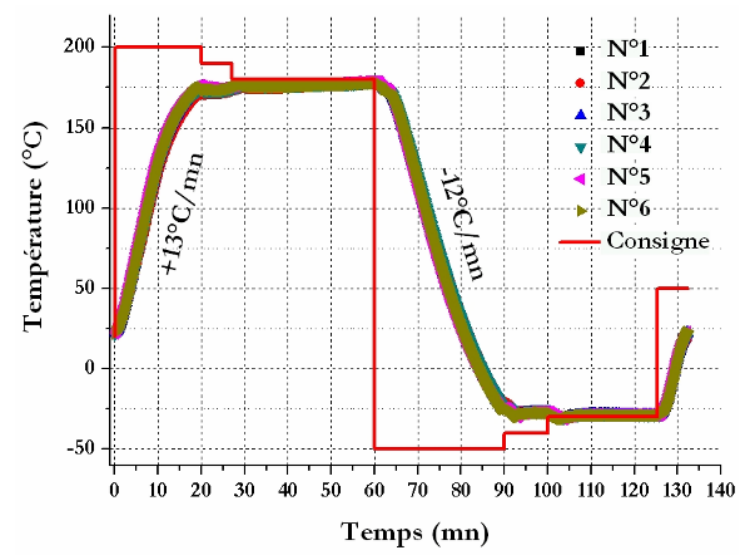

Figure 2. Température de référence, profil de température et contrôle de l'uniformité de la température dans l'enceinte

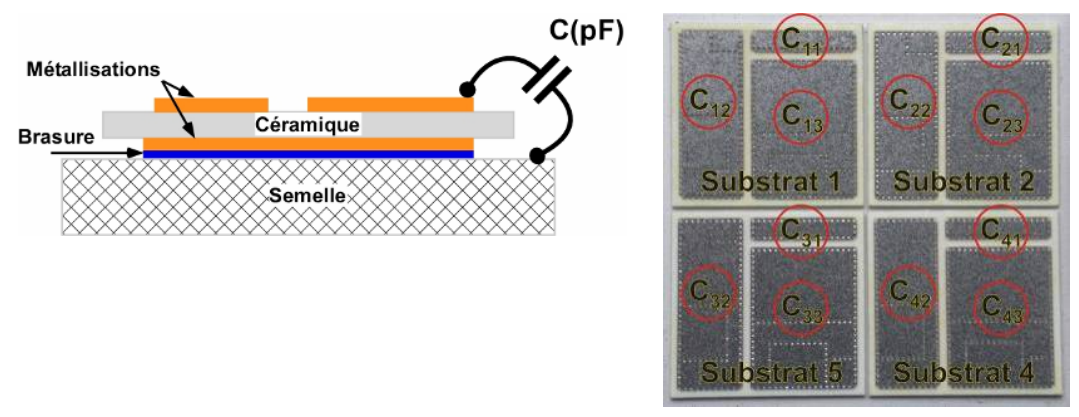

Figure 4. Méthode d'évaluation de l'intégrité du substrat céramique par mesure de la capacité, et zones d'évaluation des capacités pour le VT2.

\section{Défaillance de la céramique}

Les différences des coefficients de dilatation thermique, entre les métallisations $(\mathrm{Al}, \mathrm{Cu})$ et les céramiques ( $\mathrm{AlN}, \mathrm{Al}_{2} \mathrm{O}_{3}, \mathrm{Si}_{3} \mathrm{~N}_{4}$ ), sont à l'origine des contraintes mécaniques dans la céramique. De plus, en raison du durcissement du cuivre des métallisations des DCB, ces contraintes mécaniques augmentent régulièrement dans la céramique au cours de la répétition des cycles thermiques. Lorsque le niveau de contraintes atteint la limite de rupture de la céramique, une fracture conchoïdale apparaît dans la céramique comme le montre la figure 5. 


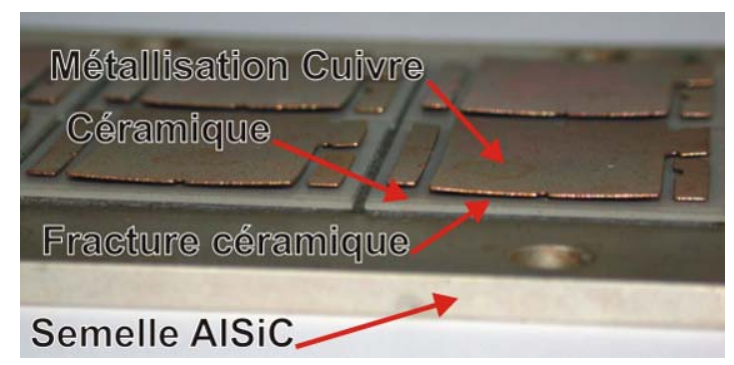

Figure 5. Fracture conchoïdale dans la céramique après 500 cycles (VT 8).

La figure 6 montre la variation relative de la capacité mesurée sur l'une des métallisations du véhicule de test $\mathrm{N}^{\circ} 7$, au cours du cyclage thermique. Le nombre de cycles nécessaire pour observer la fracture (Nf), estimé à partir de la mesure de la capacité utilisée comme indicateur de défaillance, est présenté dans le tableau 3 pour l'ensemble des véhicules de test.

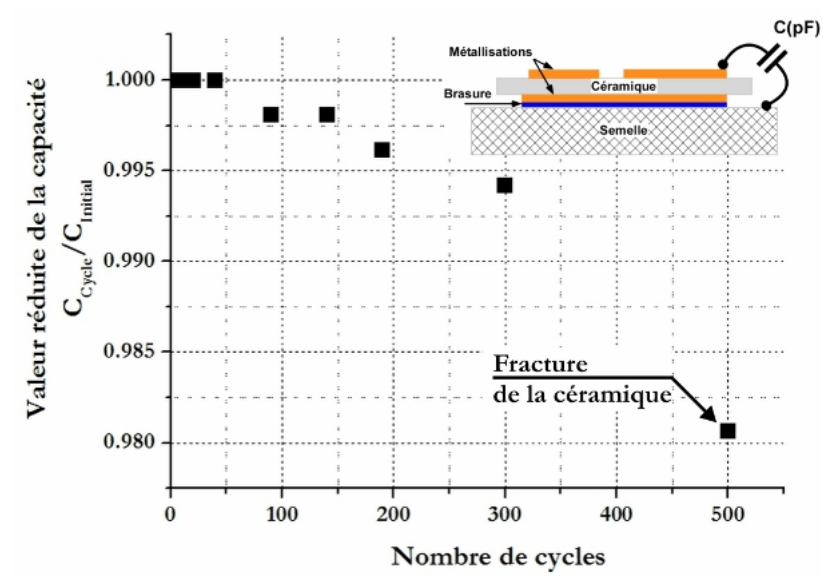

Figure 6. Variation de la valeur réduite de la capacité durant la répétition des cycles thermiques (VT 7). 
Tableau 3. Durée de vie de la céramique relevée lors de l'apparition de la fracture conchoïdale.

\begin{tabular}{|c|c|c|c|c|c|c|c|c|c|c|c|}
\hline 5 & 1 & 2 & 3 & 4 & 5 & 6 & 7 & 8 & 9 & 10 & 11 \\
\hline 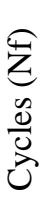 & $\begin{array}{l}\stackrel{P}{\unlhd} \\
\stackrel{1}{V} \\
\stackrel{8}{\wedge} \\
\wedge\end{array}$ & $\begin{array}{l}\stackrel{8}{尺} \\
\wedge\end{array}$ & 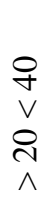 & 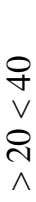 & 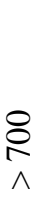 & $\begin{array}{l}\stackrel{\bigodot}{\curvearrowright} \\
\wedge\end{array}$ & \begin{tabular}{l}
8 \\
D \\
v \\
8 \\
8 \\
\} $\\
{\wedge}$ & $\begin{array}{l}8 \\
\vee \\
\text { ○े } \\
\wedge \\
\wedge\end{array}$ & $\begin{array}{l}\stackrel{尺}{尺} \\
\wedge\end{array}$ & $\begin{array}{l}\stackrel{尺}{尺} \\
\wedge\end{array}$ & $\underset{\wedge}{\stackrel{尺}{尺}}$ \\
\hline
\end{tabular}
\end{tabular}

Les résultats montrent clairement qu’une épaisseur élevée de métallisation n’est pas adaptée pour des contraintes thermiques aussi sévères que celles imposées ici. En effet, la durée de vie du substrat DCB AlN, avec 300 $\mu \mathrm{m}$ d'épaisseur de cuivre, est inférieure à 40 cycles. Considérant la même nature de céramique (AlN), nous observons clairement qu'une réduction de l'épaisseur du cuivre permet une augmentation significative de la durée de vie. Cette première conclusion confirme les résultats déjà obtenus par Curamik [6]. Les résultats sont illustrés à la figure 7 pour

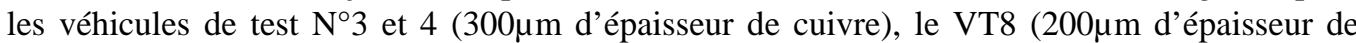
cuivre) et le VT7 (127 $\mu \mathrm{m}$ d'épaisseur de cuivre). Pour ce dernier cas, la durée de vie peut être améliorée d'un facteur proche de dix (entre 300 et 500 cycles) en comparaison avec les

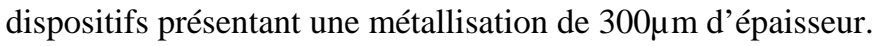

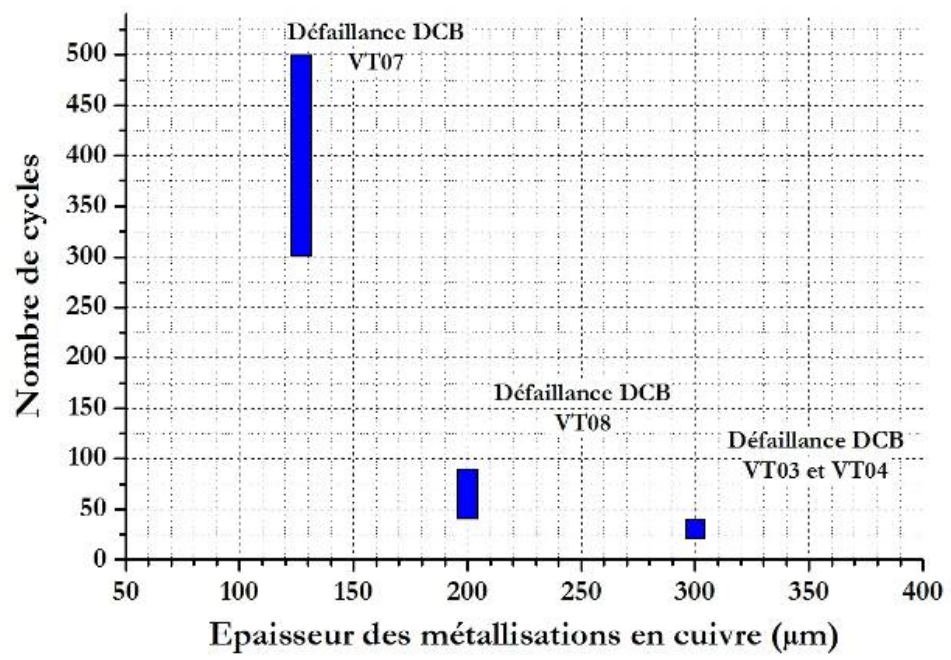

Figure 7. Influence de l'épaisseur de la métallisation sur la durée de vie du substrat DCB. 
Quelques un des véhicules de test ne présentent toujours pas de défaillance après plus de 700 cycles (arrêt provisoire des tests). C'est le cas pour le DCB présentant des métallisations en cuivre avec dimples [6], ainsi que pour les deux substrats AMB, le DAB et le substrat céramique $\mathrm{Si}_{3} \mathrm{~N}_{4}$.

Dans le but d'accroître la durée de vie des substrats céramiques DCB, les contraintes mécaniques dans la céramique, induites par le durcissement du cuivre des métallisations lors de la répétition des cycles de température, doivent être diminuées, et/ou on doit utiliser des céramiques présentant une résistance mécanique plus élevée.

La présence de dimples, à la périphérie des métallisations, permet de réduire les contraintes mécaniques induites dans la céramique. Comme présenté pour le VT2, l'effet des dimples est significatif, avec l'absence de défaillance relevée après plus de 700 cycles thermiques de forte amplitude. Les dimples agissent ainsi comme une réduction localisée de l’épaisseur de cuivre.

Les substrats AMB DAB sur céramique AlN (VT8) semblent être là aussi une solution technologique très intéressante dans le cas de sollicitations thermiques très contraignantes. Malgré un CDT élevé ( $23 \mu \mathrm{m} / \mathrm{mK}$ ), l'aluminium présente une limite élastique et un taux de durcissement bien plus faible que le cuivre [7]. Ainsi, l'augmentation de la contrainte dans la céramique, au cours de la répétition des cycles thermiques, est réduite dans le cas de métallisation en aluminium, ce qui augmente de manière très significative la durée de vie du substrat.

Finalement, l'utilisation de céramique $\mathrm{Si}_{3} \mathrm{~N}_{4}$ est une solution également très attractive pour améliorer la durée de vie du substrat isolant dans le cas de cycles thermiques de grande amplitude. La limite de rupture mécanique de la céramique $\mathrm{Si}_{3} \mathrm{~N}_{4}$ est bien plus élevée que celle de l'AlN permettant d'éviter l'apparition de fracture conchoïdale. Ainsi, aucune défaillance n'a été relevée pour la solution $\mathrm{AMB}$, avec une céramique $\mathrm{Si}_{3} \mathrm{~N}_{4}$ (VT 5), après plus de 700 cycles. Ce dernier résultat démontre clairement l'intérêt d'utiliser des céramiques présentant une limite de rupture mécanique élevée.

\section{Délaminage de la brasure}

L'initiation et la propagation de la fracture dans la brasure, au cours de la répétition des cycles thermiques, est principalement le résultat de la différence des coefficients de dilatation thermique entre la semelle et l'ensemble qui compose le substrat isolant (métallisations et céramique).

La semelle (AlSiC) et la brasure sont identiques pour l'ensemble des véhicules de test. Les différents composants testés nous permettent d'observer l'influence de la nature de la céramique et des métallisations sur la durée de vie de la brasure.

L’étude expérimentale réalisée peut ainsi permettre de comparer l'apparition du délaminage des brasures pour les différents véhicules de test. A titre d'exemple, les résultats de l'analyse 
acoustiques présentés dans la figure 8 rendent compte de l'initiation puis de la propagation du délaminage dans la brasure du VT6.

On constate pour ce véhicule de test particulier que le délaminage de la brasure apparaît préférentiellement dans l'angle inférieur et à droite. L'analyse acoustique en mode B-Scan, présentée dans la figure 9, permet d'évaluer qualitativement l'épaisseur de la brasure sous chacun des substrats céramiques du VT6. Cette analyse montre clairement que le délaminage de la brasure s'initie prioritairement dans la zone où la brasure présente la plus faible épaisseur, résultat déjà bien connu $[8,9]$.

En raison de la dépendance significative de la durée de vie de la brasure avec l'épaisseur de la brasure, il pourra être relativement difficile de comparer l'ensemble des véhicules de test. Néanmoins, nous pouvons comparer l'influence de la surface des substrats sur l'intégrité de la brasure, sous réserve d'un contrôle de l'épaisseur de la brasure là où s'initie le délaminage.

Un module préalablement testé dans des conditions de contraintes similaires [3], avec des dimensions de substrats élevées $\left(5.08 \times 5.08 \mathrm{~cm}^{2}\right)$, a présenté une initiation du délaminage de la brasure après seulement 90 cycles thermiques. Le VT3, avec des matériaux équivalents et une dimension des substrats plus faible $\left(2.54 \times 3.70 \mathrm{~cm}^{2}\right)$, présente quant à lui une initiation du délaminage après 300 cycles. Cette constatation démontre, en accord avec de précédentes études $[8,10]$, l'influence de la surface de la brasure, et donc celle du substrat céramique sur la tenue au cyclage thermique de la brasure.

L'épaisseur de la métallisation des substrats DCB (côté semelle) est un autre paramètre influent. La différence entre le VT7 et le VT8 est uniquement relative à l'épaisseur de la métallisation en cuivre sur une céramique AlN, respectivement de $127 \mu \mathrm{m}$ et $200 \mu \mathrm{m}$. Les résultats sont présentés dans la figure 10 , où nous pouvons observer une initiation du délaminage de la brasure du VT8 après 140 cycles seulement. Aucun délaminage n’est observé pour le VT7, présentant une métallisation d'épaisseur plus réduite, après 300 cycles.

Enfin, nous pouvons apprécier le très bon comportement du VT 2, présentant des dimples sur la métallisation inférieure du substrat. Aucune initiation de délaminage n’a été observée après 778 cycles thermiques (cf. Fig. 11). Les dimples semblent là aussi présenter le même effet que celui d'une réduction locale de l'épaisseur de la métallisation.

Ainsi, l'effet positif des dimples sur la durée de vie de l'assemblage, clairement démontré pour le cas de la fracture de la céramique, semble également s'appliquer sur la brasure, en retardant significativement l'apparition du délaminage. 


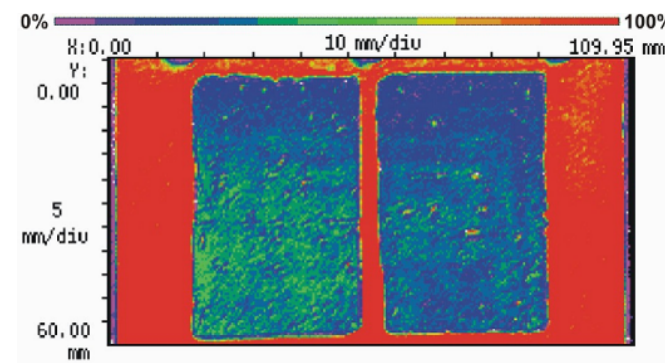

Avant le cyclage.

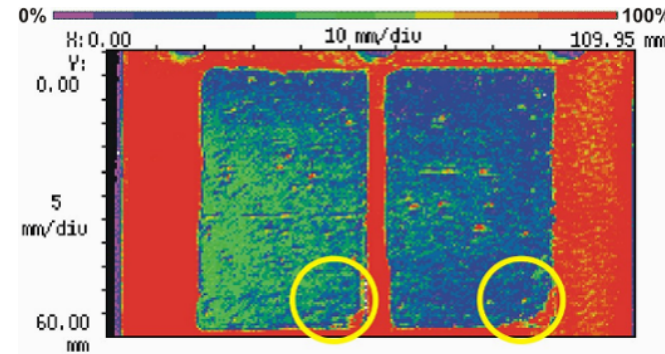

Après 190 cycles thermiques.

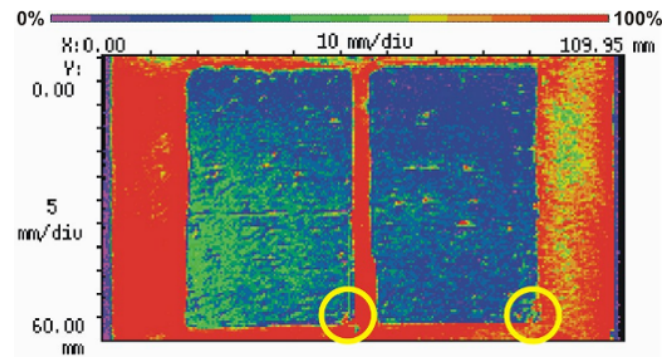

Après 40 cycles thermiques.

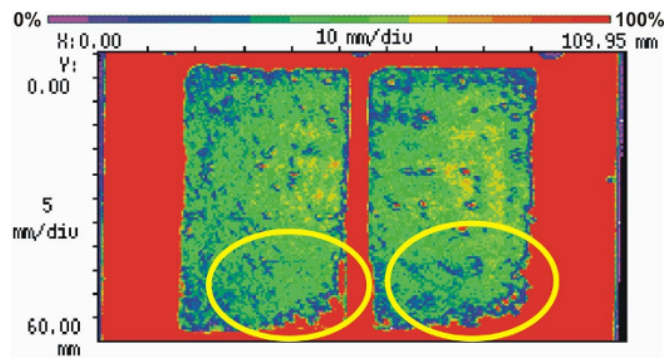

Après 500 cycles thermiques.

Figure 8. Evaluation du délaminage de la brasure pour le VT6 par microscopie acoustique

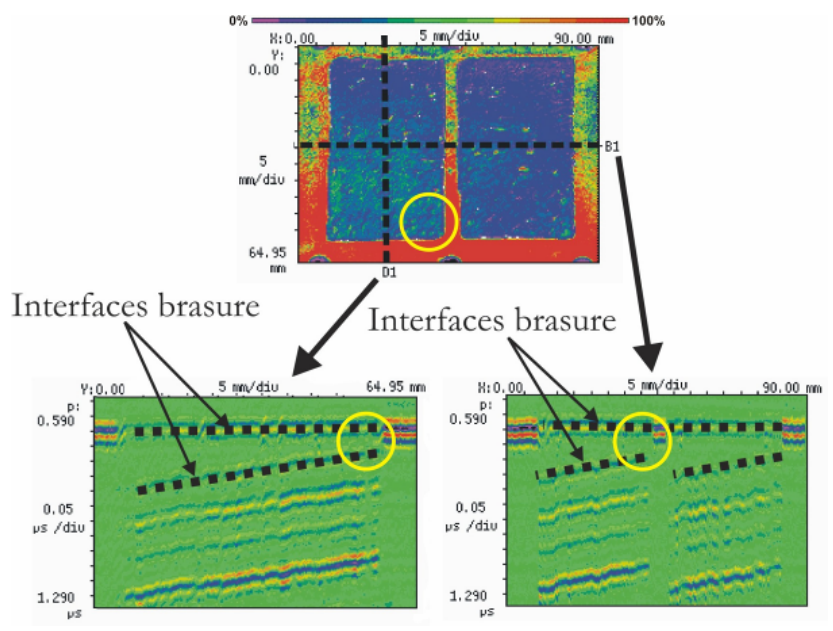

Figure 9. Variation de l'épaisseur de la brasure. 


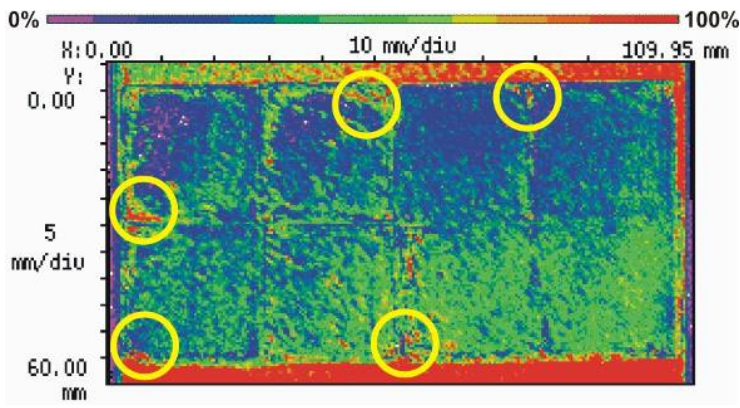

VT8 après 140 cycles (Cu : 200 нm).

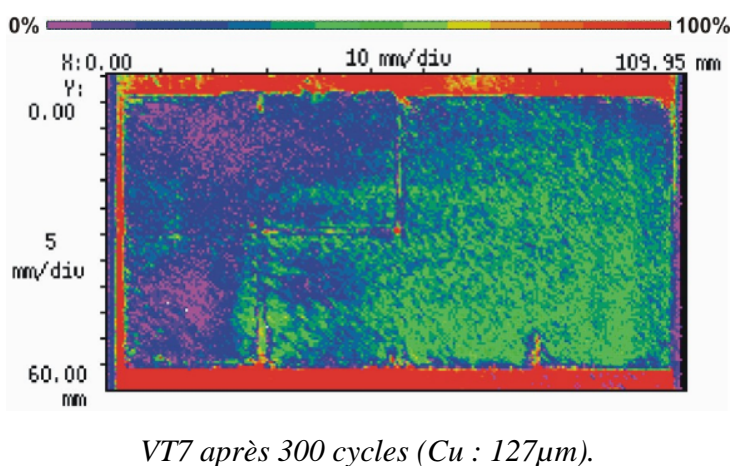

VT7 après 300 cycles (Cu : 127 $\mu$ m).

Figure 10. Influence de l'épaisseur de la métallisation sur le nombre de cycles avant initiation du délaminage.
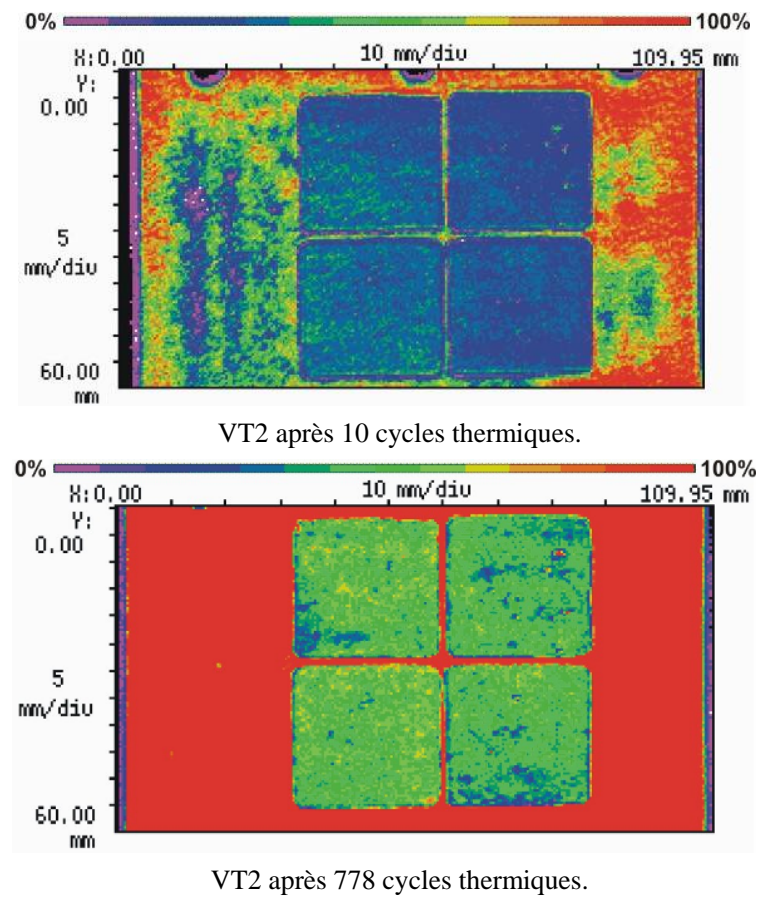

Figure 11. Effet positif de la présence de dimples sur la métallisation inférieure du substrat DCB. 


\section{Conclusion}

Cette étude présente principalement des résultats expérimentaux sur la durée de vie de différentes technologies d'assemblage de puissance soumis à des cycles thermiques de grande amplitude et à haute température.

Le comportement de différents véhicules de test mettant en œuvre des solutions technologiques différentes a été étudié. Deux principaux modes de défaillance ont été investigués, la fracturation de la céramique des substrats (DCB, $A M B S_{3} \mathrm{~N}_{4}$ et $\mathrm{DAB}$ ), et le délaminage de la brasure située entre la semelle AlSiC et le substrat céramique.

La fissure conchoïdale de la céramique provient du durcissement des métallisations et est initiée lorsque le niveau des contraintes mécaniques dépasse le seuil de rupture de la céramique. Nous avons pu montrer, dans le cas de substrats DCB (métallisations en cuivre), que la durée de vie du substrat céramique était considérablement augmentée en réduisant l'épaisseur des métallisations en cuivre. Ainsi, pour améliorer la durée de vie d'un tel assemblage, l'épaisseur de la métallisation doit être réduite au mieux, en adéquation avec le niveau de densité de courant acceptable pour les conducteurs réalisés. Les dimples, placés à la périphérie des métallisations, agissant comme une réduction locale de l'épaisseur des métallisations en cuivre, ce qui conduit à augmenter la durée de vie des substrats céramique.

Des résultats analogues (pas de défaillance après plus de 700 cycles $-30^{\circ} \mathrm{C} /+180^{\circ} \mathrm{C}$ ) sont obtenus avec le substrat céramique type DAB. La limite élastique de l'aluminium est plus faible que celle du cuivre, mais surtout, l'écrouissage de l'aluminium est négligeable (taux de durcissement de l'aluminium bien plus faible que celui du cuivre). Ainsi, la répétition des cycles n'entraîne pas d'augmentation des contraintes reportées dans la céramique comme cela pouvait être le cas avec des métallisations en cuivre.

La céramique Si3N4 semble également très attractive en raison de sa résistance mécanique élevée. Malgré le durcissement du cuivre, et donc l'augmentation des contraintes dans la céramique lors de la répétition des cycles de température, nous n'avons pas constaté avec une céramique Si3N4 d'apparition de fracture après plus de 700 cycles également.

Nous avons également cherché lors de cette étude à étudier le délaminage de la brasure, induite par les différences de coefficient de dilatation thermique entre la semelle et les matériaux du substrat céramique. Nous avons pu constater que la durée de vie de la brasure était considérablement augmentée pour des substrats céramiques de plus faibles dimensions. Mais l'initiation du délaminage (et donc la durée de vie de la brasure) semble également dépendre fortement de l'épaisseur de la métallisation du substrat céramique. Les dimples agissant comme une réduction localisée de l'épaisseur du cuivre des DCB leur effet est donc également extrêmement bénéfique sur la durée de vie de la brasure. 


\section{Bibliographie}

[1] W. Wondrack, "Physical Limits and Lifetime Limitations of Semiconductor Devices at High Temperatures", Microelectronics Reliability, June-July 1999, Vol. 39 (6-7), p. 1113-1120.

[2] P. McCluskey and al., "Packaging of Power Electronics for High Temperature Applications", Advancing Microelectronics, Jan./Feb. 1998, p. 19-24.

[3] G. Mitic, R. Beinert, and al., "Reliability of AlN Substrates and their Solder Joints in IGBT Power Modules", Microelectronics Reliability, 1999, Vol. 39, p. 1159-1164.

[4] J. Schulz-Harder, "Advantages and New Development of direct Bonded Copper Substrates", Microelectronics Reliability, 2003, Vol. 43, p. 359-365.

[5] L. Dupont \& al. "Electrical Characterizations and evaluation of thermo-mechanical stresses of a power module dedicated to high temperature applications“ proceedings of EPE 2005, Dresde, September 2005.

[6] J. Schulz-Harder, “Direct Copper Bonded Substrates for Semiconductor Power Devices”, Curamik.

[7] Y. Nagatomo and T. Nagase, "The Study of the "Power Modules with High Reliability for EV Use", Conference 17th EVS, Montreal, October 2000.

[8] E. Woirgard, Thèse, "Contribution à l'étude d'une intégration hybride adaptée à l'électronique automobile", 1992, Bordeaux 1.

[9] J. Yamada, T. Simizu, et al., "the latest High Performance and High Reliability in New packages with Conventional Pin Layout", Conférence PCIM, 2004.

[10] M. Ciappa, "Selected Failure Mechanisms of Modern power Modules" , Microelectronics Reliability, Vol. 42, 2002, pages : 653-667. 